\title{
Capacidad germinativa de Poaceae nativas de las sierras de Córdoba (Argentina) afectadas por incendios
}

\author{
Martinat, J. E. y Gil, S. P.
}

DOI 10.31047/1668.298x.v38.n2.28090

\begin{abstract}
RESUMEN
En ambientes serranos de Córdoba (Argentina) donde predomina la actividad turística y ganadera basada en especies forrajeras nativas, los incendios que afectan la zona comprometen la recuperación del paisaje natural. El objetivo de este trabajo fue evaluar la capacidad germinativa de Eustachys retusa y Schizachyrium condensatum, forrajeras nativas, sometidas a tratamientos pregerminativos y a factores del fuego. A sus semillas se les aplicaron tres repeticiones de los siguientes tratamientos: nitrato de potasio, frío, humo y choques térmicos. Se evaluó la germinación durante 21 días. Se observó un incremento favorable de la germinación en los tratamientos de $90^{\circ} \mathrm{C}$ para $S$. condensatum y una disminución en la germinación de E. retusa en los tratamientos de frío y $120^{\circ} \mathrm{C}$ durante $10 \mathrm{~min}$. En el resto de los tratamientos realizados no hubo diferencias significativas con el testigo. En ninguno de los tratamientos S. condensatum alcanzó el $50 \%$ de germinación, mientras que $E$. retusa resultó siempre mayor al $70 \%$. Se concluye que las semillas de $E$. retusa y $S$. condensatum toleran temperaturas de incendios rasantes o moderados y son capaces de germinar a $120^{\circ} \mathrm{C}$. E. retusa presenta ventajas para colonizar sitios quemados de las serranías cordobesas.
\end{abstract}

Palabras clave: propagación, choque térmico, humo, Eustachys retusa, Schizachyrium condensatum

Martinat, J. E. and Gil, S. P. 2021. Reproductive capacity of native Poaceae from the mountains of Córdoba (Argentina) affected by fires. Agriscientia 38 (2): 41-51

\section{SUMMARY}

In mountain environments of Córdoba (Argentina), where tourist activity predominates and livestock activity is based on native species, the fires that affect the area compromise the recovery of the natural landscape. The objective of this work was to evaluate the germination capacity of Eustachys retusa and Schizachyrium condensatum, native forages, subjected to pre-germination 
treatments and fire factors. Three repetitions were performed for each seed treatment: potassium nitrate, cold, smoke and heat shock tests. Germination was assessed for 21 days. A favorable increase in germination was observed in the $90^{\circ} \mathrm{C}$ treatments for $\mathrm{S}$. condensatum and a decrease in germination of E. retusa in cold and $120^{\circ} \mathrm{C}$ for 10 min treatments. In the rest of the treatments carried out there were no significant differences with the control. In none of the treatments S. condensatum reached $50 \%$ germination, while E. retusa was always higher than $70 \%$. We conclude that the seeds of E. retusa and $S$. condensatum tolerate low or moderate fire temperatures and are capable of germinating at $120^{\circ} \mathrm{C}$. E. retusa has advantages for colonizing burned sites in the Cordoba highlands.

Key words: propagation, heat shock, smoke, Eustachys retusa, Schizachyrium condensatum

Martinat, J. E. (ORCID: 0000-0002-8456-4879) y Gil, S. P. (ORCID: 0000-0002-7440-6172): Universidad Nacional de Córdoba, Facultad de Ciencias Agropecuarias, Cátedra de Botánica Agrícolal y II, Laboratorio de Microscopía Óptica, Herbario ACOR. Félix A. Marrone 746, 5000 Córdoba. Correspondencia a: jmartinat@agro.unc.edu.ar

\section{INTRODUCCIÓN}

El fuego es un factor de alto impacto que influye en la estructura y función de numerosos ecosistemas del mundo y actúa como un agente de selección de la persistencia y regeneración de distintas especies (Keith et al., 2002; Lamont y Downes, 2011; Pausas y Schwilk, 2012; Co du Toit et al., 2015). En Argentina, el Bosque Chaqueño está profundamente modificado y presenta un encadenamiento de áreas deforestadas, que responde mayormente a la acción del fuego y al avance de la frontera agropecuaria (Zak et al., 2004; Preliasco y Miñarro, 2016; Torrella et al., 2018). Particularmente en las Sierras Chicas de Córdoba (altura máxima 1980 m s.n.m.), la mayor ocurrencia de incendios se debe principalmente a la actividad antrópica, y afecta a matorrales y pastizales debido a sus características de inflamabilidad y a su fisonomía abierta, que favorece la dominancia de especies herbáceas y la propagación del fuego (Carbone et al., 2017; Argañaraz et al., 2018). Evaluaciones florísticas postincendio en las sierras cordobesas, han revelado que la frecuencia del fuego produce cambios en la estructura de la vegetación, en la riqueza y en la composición florística (Cabido y Zak, 1999; Verzino et al., 2005; Carbone et al., 2017). Entre las especies forrajeras de Poaceae, que representan el $20 \%$ del total de los taxones censados en las zonas afectadas por incendios (Fuentes et al., 2010), se destacan Eustachys retusa (Lag.) Kunth y Schizachyrium condensatum (Kunth) Nees (Privitello et al., 2010; Fuentes et al., 2011; Giorgis et al., 2011). Además, poseen carácter atractivo para su potencial uso ornamental en áreas serranas (Sánchez de Lorenzo-Cáceres, 2006; Arancibia, 2007; Martinat et al., 2019), lo que contribuiría a la revalorización de la flora nativa.

Los estudios sobre la capacidad y calidad de regeneración de especies autóctonas de las sierras de Córdoba, son relevantes para predecir las posibilidades de recuperación en ambientes afectados por el fuego (Arcamone y Jaureguiberry, 2018). La regeneración de las especies vegetales en ambientes sujetos a disturbios depende, entre otros, de la ubicación de las semillas en el suelo, su viabilidad y capacidad de germinación (Buhk y Hensen, 2006; Buhk et al., 2007; Lamont y Downes, 2011; Giambastiani, 2019). La mayoría de las estrategias utilizadas para mantener la biodiversidad en los ecosistemas propensos a incendios requieren una buena comprensión de la relación entre los efectos del fuego directo y la germinación (Baeza y Roy, 2008; Ribeiro et al., 2013). La falta de disponibilidad de semillas y el desconocimiento de la capacidad germinativa de semillas de herbáceas nativas se presentan como limitantes para su uso en restauración y en general se recurre a especies exóticas con protocolos 
de germinación conocidos que actúan como otro factor de alteración del ambiente natural y compiten con las nativas. En este sentido, se sabe que $E$. retusa tiene un alto poder germinativo y que se resiembra naturalmente (Cáceres et al., 2018) y que $S$. condensatum tiene un banco de semillas persistente a largo plazo (Ferri et al., 2009). Sin embargo, se desconoce la capacidad germinativa de las semillas de estas especies luego de ser sometidas a tratamientos pregerminativos, que podrían emplearse para lograr altos porcentajes de germinación en programas de siembra y restauración de ambientes degradados. Tampoco se conoce la tolerancia de sus semillas a las altas temperaturas y al humo (Martinat, 2012; Martinat et al., 2017), información que contribuiría al conocimiento científico y predicción de su potencial uso en restauración natural. Las temperaturas y el humo alcanzados durante los incendios pueden promover la germinación de las semillas almacenadas en el suelo o romper la latencia, permitiendo posteriormente la imbibición del agua y la germinación de las semillas en el suelo, cuando las condiciones en el ambiente son las adecuadas (Veneciano, 2006; Downes et al., 2010; Moreira et al., 2010; Tavsanoglu, 2011; Chou et al., 2012; Burger et al., 2018; Keeley y Pausas, 2018). En algunas especies de Poaceae, las cubiertas de las cariópsides, conocidas como lemma y pálea, son las causantes de latencia debido a su dureza e impermeabilidad que puede ser debilitada con altas temperaturas (Reeder, 1977; Ernst et al.,1991; Schrauf et al., 1998).

En función de lo expuesto, se planteó el objetivo de evaluar la capacidad germinativa de E. retusa y $S$. condensatum sometidas a tratamientos pregerminativos recomendados por la Asociación Internacional de Ensayos de Semillas (International Seed Testing Association [ISTA], 2019) y a condiciones de choques térmicos y humo que recrean los efectos del fuego. Este trabajo contribuirá a la comprensión de los procesos ecológicos reproductivos y de establecimiento de especies en estos ambientes postfuego y aportará herramientas para la elaboración de modelos predictivos brindando fundamentos para un manejo sustentable.

\section{MATERIALES Y MÉTODOS}

\section{Especies de estudio}

Eustachys retusa (Lag.) Kunth (Chloridoideae), conocida como "pata de gallo", es una especie perenne con rizomas cortos, de $25-80 \mathrm{~cm}$ de altura, de crecimiento estival. Posee hojas con lámina plana de 6-10 mm de ancho y vaina lateralmente comprimida, glabra, menores que los entrenudos. La inflorescencia es de $3-10 \mathrm{~cm}$ de longitud, formada por dos a numerosos racimos unilaterales verticilados (Zuloaga et al., 2012). Es una buena forrajera resistente al pastoreo y, si bien proporciona poca cantidad de forraje, es un pasto de mediana a alta palatabilidad (Aguilera et al., 1998). También podría utilizarse como planta ornamental (Martinat et al., 2019).

Schizachyrium condensatum (Kunth) Nees (Panicoideae), conocida como "pasto colorado", es una hierba perenne, erecta, de $35-120 \mathrm{~cm}$ de altura y crecimiento estival. Posee lámina generalmente plana de 2,7-4,5 $\mathrm{mm}$ de ancho y vaina convoluta con márgenes rojizos. La inflorescencia es una panoja corimbiforme (Peichoto, 2010; Zuloaga et al., 2012). Posee un rebrote tierno que es útil como forraje para el ganado, al momento de la floración la planta pierde calidad debido a la elevada cantidad de cañas que posee y se convierte en paja dura casi inútil como forrajera (Southwest Environmental Information Network, 2019). La forma de la planta, su vistosidad en estado reproductivo y color de inflorescencia la posicionan con altas posibilidades de uso en parques y jardines (Martinat et al., 2019).

\section{Material vegetal}

Durante los meses de febrero y marzo de 2018 y 2019 se recolectaron semillas maduras del banco de semillas aéreo de E. retusa y $S$. condensatum de poblaciones ubicadas en ladera oriental de las Sierras Chicas que se caracterizan por un relieve de sierras bajas (500-1980 m s.n.m.) con orientación montañosa norte-sur. El clima es de dominio semihúmedo en verano con tendencia a semiseco en invierno, con temperaturas mínimas y máximas promedios entre 10 y $25^{\circ} \mathrm{C}$ (Capitanelli, 1979). La precipitación varía entre los $700-900$ mm anuales, con grandes variaciones interanuales (entre 600 y 1500 mm) (Carmen y Ana María Malanca, comunicación personal, 10 de marzo de 2017) y se concentra en la estación cálida, delimitando un período de déficit hídrico invernal (Giorgis et al., 2013; Carbone et al., 2017). Está comprendida en la región fitogeográfica Distrito Chaqueño, degradada por antiguos incendios, entre otros factores (Argañaraz et al., 2018). La zona de estudio pertenece al departamento Colón, Estancia La Estancita (Córdoba, Argentina, $31^{\circ}$ 07' 10,0" S, 64' 23' 04,9" O) con antecedentes de incendios del año 2015 y comprende 500 ha aproximadamente (Figura 1). De cada especie y 
por cada temporada de recolección, se conformó un pool de semillas proveniente de individuos al azar de la zona de recolección, procurando una muestra representativa que contemple la variabilidad existente. Se preservaron en bolsas de papel, a temperatura ambiente, en oscuridad y lugar seco, hasta el momento de su utilización. Ejemplares completos de cada especie se herborizaron para su documentación en el Herbario ACOR de la Facultad de Ciencias Agropecuarias de la Universidad Nacional de Córdoba.

Con la finalidad de simular lo que ocurre en la naturaleza, para los ensayos se consideró la unidad natural de dispersión como semilla y se aseguró la existencia de una sola cariópside mediante microscopio estereoscópico Carl Zeiss. En E. retusa la semilla es representada por la espiguilla separada de sus glumas y en $S$. condensatum por el par de espiguillas y el artejo del raquis.

\section{Tratamientos pregerminativos recomendados por las Asociación Internacional de Ensayos de Semillas}

Las Poaceae forrajeras normalmente utilizadas para reforestar áreas serranas afectadas por incendios, son especies exóticas que se ofrecen comercialmente con alto poder germinativo, avalado con análisis que respetan protocolos establecidos por las Reglas Internacionales de
Análisis de Semillas (ISTA, 2019) donde además se indica, para cada especie estudiada, tratamientos previos la germinación que deben realizarse para lograr mayores porcentajes germinativos. La utilización de dichas especies resulta perjudicial debido a que actúan como otro factor de alteración del ambiente natural y compiten con las nativas, alterando el equilibrio (Halvorson y Guertin, 2003). Sin embargo, los protocolos establecidos por ISTA para dichas especies, orientan la evaluación de la capacidad germinativa de especies nativas. Es por ello que la calidad de semillas se evaluó mediante la prueba de poder germinativo sugerida por las normas ISTA (2019) para especies taxonómicamente cercanas a las estudiadas (Eragrostis de la subfamilia Chloridoideae, Schizachyrium scoparium de Panicoideae). Además, las temperaturas recomendadas son concordantes con las temperaturas del momento del año en que se concentran las precipitaciones en la zona de estudio, tal como lo concluido por Funes et al. (2009).

Se utilizaron semillas con 18 meses de almacenamiento. Para $S$. condensatum se repitieron los tratamientos con semillas de 3 meses de almacenamiento, del segundo año de recolección, debido a la nula germinación del anterior.

Se realizó para cada taxón, previo a la germinación, un tratamiento con nitrato de potasio y un tratamiento con frío. Para el tratamiento con

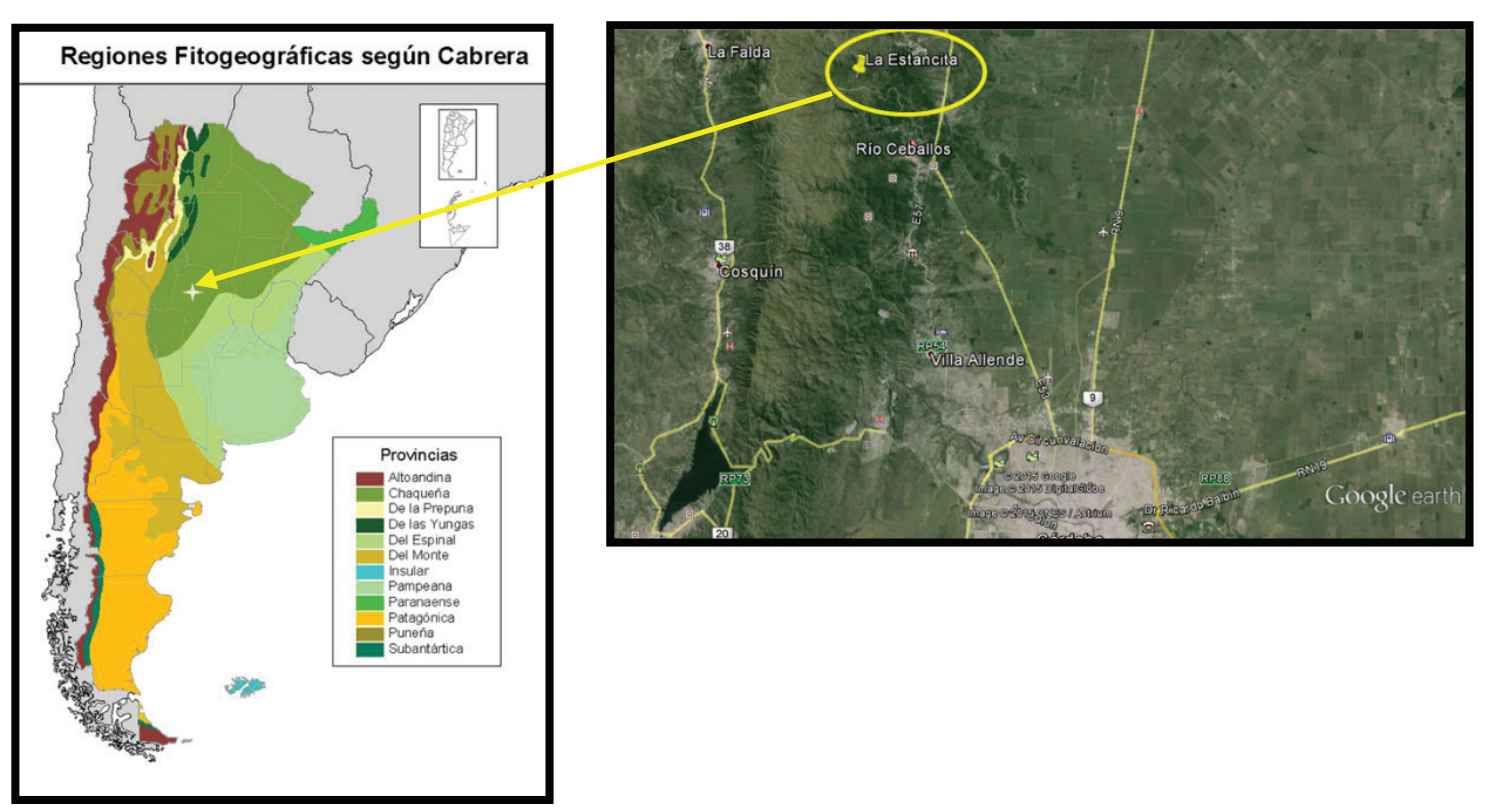

Figura 1. Ubicación de la zona de recolección del material. Estancia La Estancita, Departamento Colón, Córdoba, Argentina 
nitrato de potasio, se embebió papel Whatman con una solución al $2 \%$ y se colocó en las bandejas de germinación. El tratamiento de frío se realizó en heladera a $8^{\circ} \mathrm{C}$ durante 7 días. Se realizaron tres repeticiones de 25 semillas cada uno. Las semillas de todos los tratamientos, más un grupo de semillas testigo sin tratar, se sembraron en bandejas de germinación, se cubrieron con nylon y se dispusieron en cámara de germinación en el Laboratorio de Análisis de Semillas de la Facultad de Ciencias Agropecuarias de la Universidad Nacional de Córdoba con $8 \mathrm{~h}$ de luz a $30^{\circ} \mathrm{C}$ y $16 \mathrm{~h}$ de oscuridad a $20^{\circ} \mathrm{C}$. Se realizaron tres repeticiones para cada tratamiento. Se observó la germinación cada dos días y se consideró hasta el día 21 desde la siembra para su análisis, momento en el que se observó estabilidad en la curva de germinación. Se consideró la germinación fisiológica sensu stricto, es decir, $2 \mathrm{~mm}$ de radícula para unificar la evaluación con los ensayos de choque térmico y humo, siguiendo las experiencias de Hanley et al. (2003), Overbeck et al. (2006) y Paula y Pausas (2008)

\section{Ensayos de choque térmico y humo, simulando los efectos del fuego}

Para simular las condiciones naturales, las semillas no recibieron pretratamiento y de cada especie se tomaron semillas al azar, distribuidas en 3 repeticiones de 25 semillas para cada tratamiento. Se utilizaron semillas con 8-9 meses de almacenamiento, coincidiendo con la edad que normalmente alcanzan las semillas al momento de ocurrencia de los incendios y posteriores lluvias. Los tratamientos aplicados fueron:

I) Choque térmico en estufa eléctrica. En función de los estudios realizados previamente por otros autores (Ruyle et al., 1988; Hanley et al., 2003; Overbeck et al., 2006; Paula y Pausas, 2008; Bolin, 2009), se seleccionaron tres temperaturas, 60,90 y $120{ }^{\circ} \mathrm{C}$, y dos tiempos de aplicación, 5 y $10 \mathrm{~min}$. Las semillas se dispusieron en cajas de Petri de vidrio de $9 \mathrm{~cm}$ de diámetro, sin tapa, distantes de los bordes y asegurando uniformidad en su distribución.

Las temperaturas seleccionadas son concordantes con las registradas en incendios en ecosistemas argentinos, tal como lo señalado por Kunst et al. (2003) y Lupi (2015) quienes indicaron que, en fuegos de pastizales, las temperaturas máximas promedio observadas en el primer centímetro de suelo son de $60-80^{\circ} \mathrm{C}$ para distintas clases de combustibles finos, mientras que los cambios a mayor profundidad son despreciables.
En fuegos de combustibles gruesos, a $2,5 \mathrm{~cm}$ del suelo las temperaturas variaron entre 90 y $195^{\circ} \mathrm{C}$, alcanzando un valor de $50^{\circ} \mathrm{C}$ a los $5 \mathrm{~cm}$.

Posteriormente, con los seis tratamientos más el testigo de cada especie, se llevó a cabo el ensayo de germinación con la misma metodología que la descripta para los tratamientos pregerminativos.

II) Expuestas durante 6 min a humo proveniente de la quema de material vegetal seco de especies recolectadas del lugar de estudio, que crecen conjuntamente a las que se evalúan. Se empleó un aplicador de humo tipo Fume 2000, creado a partir de lo descripto en la bibliografía consultada (Casal et al., 2001; Iglesia Rodríguez, 2010).

A continuación, se llevó a cabo el ensayo de germinación con la misma metodología que la descripta para los tratamientos pregerminativos.

\section{Análisis estadístico}

Los resultados se analizaron estadísticamente mediante Modelos Lineales Generalizados Mixtos (MLGM), utilizando el paquete Ime4 (Bates et al., 2015) de $\mathrm{R}$ mediante el sistema estadístico INFOSTAT (Di Rienzo et al., 2020). Para el primer experimento se utilizó como factor nitrato de potasio y frío, para el segundo, temperatura en interacción con el tiempo, mientras que para el tercer experimento se utilizó el humo como factor. La prueba de comparación utilizada fue la de Fisher (LSD). Se siguió un diseño completamente aleatorizado.

\section{RESULTADOS Y DISCUSIÓN}

\section{Tratamientos pregerminativos}

S. condensatum manifestó una germinación nula en los tratamientos realizados con las semillas recolectadas el primer año, es por ello que se repitieron los tratamientos con la recolección del mismo año de los ensayos (2019), donde se pudo registrar germinación. Esta respuesta puede deberse a algún tipo de dormición presente en las cariópsides, o bien, pérdida de viabilidad durante el almacenamiento (Reeder, 1977; Ernst et al., 1991).

El tratamiento con frío presentó una tendencia a mayor germinación en $S$. condensatum respecto del testigo (44 y $33 \%$ respectivamente). Por otro lado, en E. retusa dicho tratamiento fue el menos exitoso (75\%), demostrando una germinación significativamente menor que el testigo (89\%). El tratamiento con nitrato de potasio reflejó 
una tendencia a incrementar el porcentaje de germinación respecto del testigo en ambas especies; sin embargo, las diferencias no fueron estadísticamente significativas (Figura 2A-B).

E. retusa superó el $70 \%$ de germinación en todos los tratamientos mientras que $S$. condensatum no alcanzó el $50 \%$ hasta el final del ensayo para ninguno de los tratamientos (Figura 2). Esta mayor emergencia de plántulas en E. retusa le daría una ventaja para colonizar áreas degradadas dentro de las Sierras Chicas de Córdoba (Giambastiani, 2019).

\section{Ensayos de choque térmico y humo}

Los tratamientos con choques térmicos con temperaturas de 60 y $90^{\circ} \mathrm{C}$ tuvieron una tendencia a incrementar la germinación de E. retusa (95 a $99 \%) ;$ sin embargo, el análisis estadístico indica que dichas diferencias no son significativas en relación al testigo (93\%). Estos resultados concuerdan con Ribeiro et al. (2013) quienes concluyeron que, en general, las altas temperaturas aplicadas no afectaron la tasa de germinación de las semillas tolerantes en diversas especies de la sabana brasilera. Además, podría explicar en parte, la alta frecuencia relativa de $E$. retusa en los censos florísticos realizados en las Sierras Chicas por Carbone et al. (2017) en los escenarios de baja frecuencia de fuego (100\%) y no quemado $(67 \%)$.

La exposición a $120^{\circ} \mathrm{C}$ durante 5 min no afectó significativamente la germinación en $E$. retusa
(92\%); sin embargo, al incrementar el tiempo de exposición a $10 \mathrm{~min}$, la germinación resultó menor que el testigo ( $77 \%$ ), mostrando diferencias estadísticamente significativas (Figura 3A). Es decir que temperaturas altas generarían una baja en la germinación solo si la exposición es por largo tiempo. Resultados similares fueron reportadas por Martinat et al. (2015) en Bouteloua curtipendula (Michx.) Torr. pero con porcentajes de germinación menores al $50 \%$.

En S. condensatum se observaron los mayores porcentajes de germinación en las exposiciones a $90^{\circ} \mathrm{C}$, tanto de 5 como de 10 min (43 y $44 \%$ respectivamente), mostrando diferencias estadísticas significativas con el testigo (24\%). Los tratamientos de $60{ }^{\circ} \mathrm{C}$, tanto de 5 como de 10 min (31 y $32 \%$ respectivamente) no mostraron diferencias significativas con el testigo en esta especie (Figura 3B). Estos resultados confirman lo indicado por Ruyle et al. (1988), Veneciano (2006), Martinat (2012) y Jewsbury et al. (2016), en relación a que la variabilidad de la respuesta depende de la especie estudiada. Asimismo, Carbone et al. (2017) en los censos florísticos realizados hallaron tres especies del género Schizachyrium diferentes a la estudiada y observaron que tanto la presencia como la frecuencia relativa en escenarios de alta frecuencia de fuego, baja frecuencia y no quemado era variable según la especie. S. condensatum tuvo una tendencia a incrementar el porcentaje de germinación en relación al testigo con exposiciones a $120^{\circ} \mathrm{C}$, independientemente del tiempo de exposición (36\% con 5 min y $31 \%$ con $10 \mathrm{~min}$ )
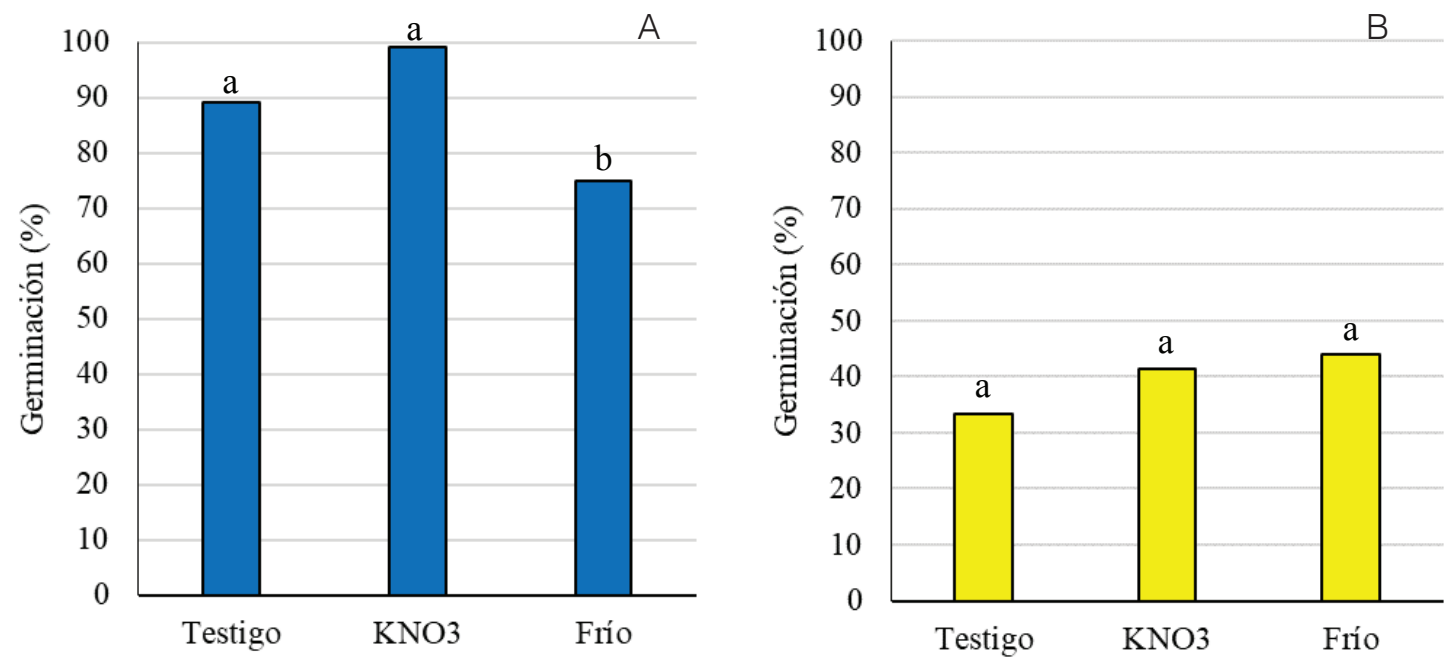

Figura 2. Porcentajes de germinación con semillas sometidas a tratamientos de frío y nitrato de potasio (KNO3):(A) Eustachys retusa,(B) Schizachyrium condensatum. Letras iguales no difieren significativamente $(p>0,05)$ 

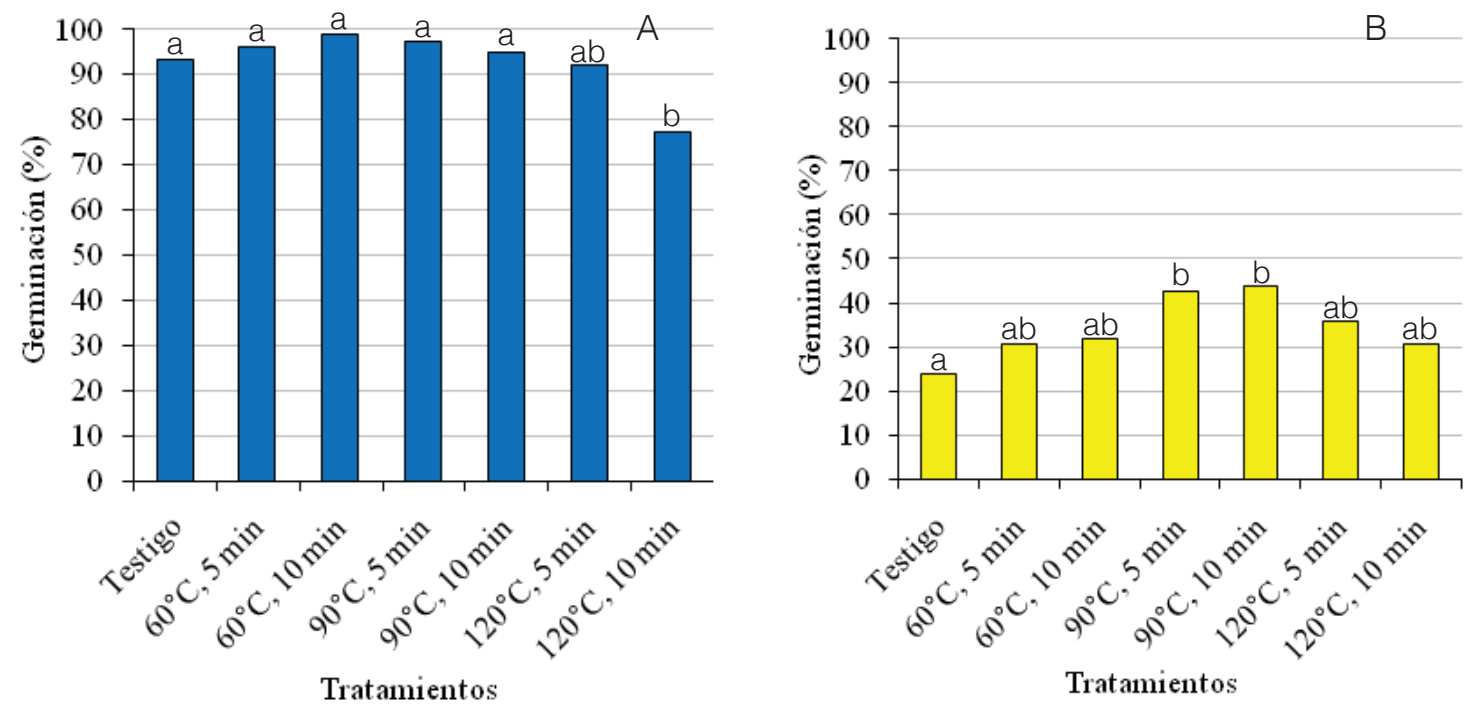

Figura 3. Porcentajes de germinación con semillas sometidas a diferentes tratamientos de choque térmico: (A) Eustachys retusa, (B) Schizachyrium condensatum. Letras iguales no difieren significativamente $(p>0,05)$

aunque la diferencia no resultó estadísticamente significativa (Figura 3B). Estos resultados podrían significar que las temperaturas elevadas, como las que se registran en los incendios, podrían favorecer a la germinación de esta especie, registrando un umbral óptimo en torno a los $90^{\circ} \mathrm{C}$.

Ambas especies estudiadas presentan ventajas respecto de Setaria lachnea (Nees) Kunth y Eragrostis tenuifolia (A. Rich.) Hochst. ex Steud., en las cuales Martinat (2012) reportó que temperaturas de $120^{\circ} \mathrm{C}$ resultan altamente perjudiciales por provocar una germinación nula.

Los incrementos en los porcentajes de germinación hallados, podrían deberse a una ruptura de dormición, tal como lo expresado por Ruyle et al. (1988), quienes expresaron que los tratamientos de choques térmicos provocados naturalmente por el fuego, son efectivos para romper dormición en cariópsides de Eragrostis lehmanniana Nees, y con Haferkamp y Jordan (1977) y Veneciano (2006) quienes indicaron que la escarificación con calor seco a $70^{\circ} \mathrm{C}$ puede romper dormición en cariópsides de E. lehmanniana con el consecuente incremento de germinación.

En los tratamientos de semillas sometidas a humo, $E$. retusa y $S$. condensatum mostraron un incremento en su germinación en relación al testigo ( $8 \%$ y $11 \%$ respectivamente) pero sin ser estadísticamente significativos (Figura 4A-B). Estos resultados pueden deberse a los hallazgos expresados por Burger et al. (2018) quienes indicaron que, en general, el humo proporciona un estímulo positivo para la germinación de muchas especies debido a la presencia de carriquines, un grupo de reguladores del crecimiento que se encuentran en el humo de la quema de material vegetal; aunque también podría deberse a otros componentes presentes en el humo (Keeley y Pausas, 2018). La tendencia al incremento en la germinación, concuerda con lo reportado por Farías et al. (2016) quienes trabajaron con otras especies de Poaceae, Aristida laevis y Erianthus angustifolius, e indicaron que el humo no afectó significativamente la germinación. Sin embargo, Arcamone y Jaureguiberry (2018), en ensayos realizados con humo y choque térmico en diez especies diferentes de Poaceae, indicaron que la respuesta es muy variable según las especies pero que todas fueron estimuladas al menos por un factor, sugiriendo que las especies estudiadas tendrían una ventaja en colonizar ambientes luego de incendios de baja a moderada intensidad.

La diferencia hallada en los testigos de los distintos tratamientos para cada especie se atribuye a la edad de las semillas ya que los ensayos se realizaron en forma consecutiva, pudiendo sufrir pérdida de viabilidad o bien algún tipo de dormición. Por otro lado, los tratamientos no resultaron eficientes en general en $S$. condensatum dado que ninguno de ellos logró superar el 50\% de semillas germinadas, lo cual podría deberse a las mismas razones mencionadas anteriormente, de lo cual no se encontraron antecedentes para esta especie. La dormición podría darse por una barrera física al pasaje de oxígeno o agua impuesta por las glumelas, o bien, por la presencia 


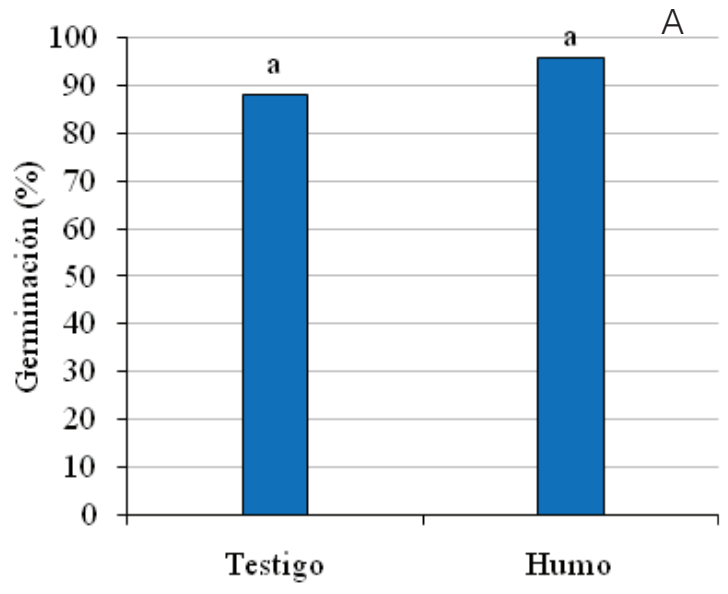

Tratamientos

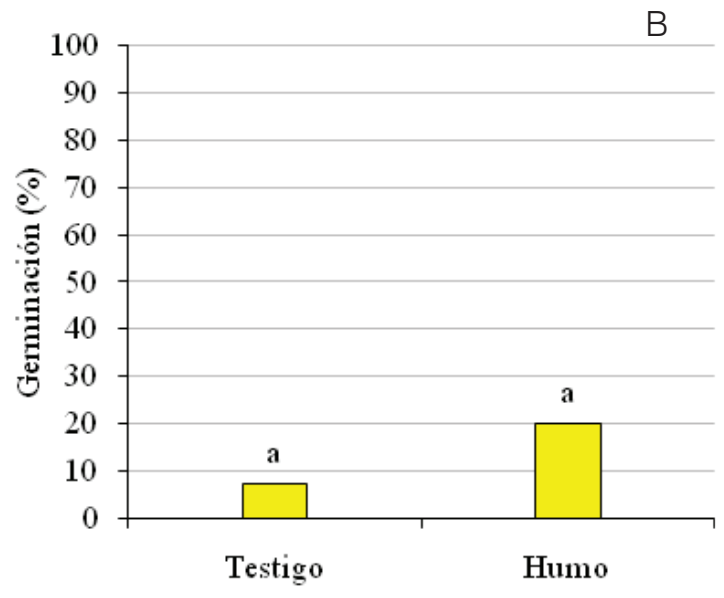

Tratamientos

Figura 4. Porcentajes de germinación con semillas sometidas a humo: (A) Eustachys retusa, (B) Schizachyrium condensatum. Letras iguales no difieren significativamente $(p>0,05)$

de inhibidores en esas coberturas, tal como lo expresado por otros autores en otras especies de Poaceae (Reeder, 1977; Ernst et al.,1991; Schrauf et al., 1998).

\section{CONCLUSIONES}

Los tratamientos de nitrato de potasio y frío no favorecen la germinación de ninguna de las dos especies, incluso el frío genera una disminución en la germinación en E. retusa.

Las semillas de E. retusa y $S$. condensatum toleran el humo y las altas temperaturas como las que se registran en incendios rasantes 0 moderados y son capaces de germinar aún después de ser expuestas a $120^{\circ} \mathrm{C}$.

Temperaturas cercanas a los $90^{\circ} \mathrm{C}$, resultan favorables para las semillas de $S$. condensatum, lo que conduciría a cambios florísticos en las comunidades afectadas por incendios de moderada intensidad. Este efecto, que naturalmente produciría el incendio sobre la germinación de $S$. condensatum, resulta más eficiente que los pretratamientos que normalmente se realizan en laboratorio (nitrato de potasio y frío) con la intención de favorecer la germinación en especies taxonómicamente cercanas a las estudiadas.

En general, E. retusa presenta un poder germinativo mayor que $S$. condensatum, lo cual, sumado a su tolerancia a las altas temperaturas y humo, representa una ventaja de esta especie para colonizar sitios quemados de las serranías cordobesas.
Estos estudios sugieren un panorama promisorio sobre las especies estudiadas y constituyen un aporte para fomentar las acciones de conservación y aprovechamiento sustentable de recursos autóctonos de interés ornamental y forrajero de Poaceae. De este modo se amplía el conocimiento sobre estas, particularmente sobre sus cualidades de reproducción en ambientes afectados por incendios.

\section{AGRADECIMIENTOS}

Los autores agradecen a la Facultad de Ciencias Agropecuarias de la Universidad Nacional de Córdoba por el financiamiento efectuado mediante el Programa de Subsidios PROIINDIT (RHCD 281/2017) y a la Secretaría de Ciencia y Tecnología de la Universidad Nacional de Córdoba, por el subsidio mediante Proyecto CONSOLIDAR (Res. SECYT $N^{\circ}$ 411/18).

Se agradece la colaboración de estudiantes y miembros de la comunidad de la Facultad de Ciencias Agropecuarias de la Universidad Nacional de Córdoba que colaboraron en las distintas actividades, especialmente al Dr. Julio Di Rienzo por sus recomendaciones para el análisis estadístico.

\section{BIBLIOGRAFÍA}

Aguilera, M. O., Steinaker, D. F., Demaría, M. R. y Ávila, A. O. (1998). Estados y transiciones de los pastizales de Sorghastrum pellitum del área medanosa central 
de San Luis, Argentina. Ecotrópicos, 11(2), 107-120. http://www.saber.ula.ve/handle/123456789/25608

Arancibia, M. (2007). Xerojardinería: explosión de color y forma con plantas nativas.

Arcamone, J. R. y Jaureguiberry, P. (2018). Germination response of common annual and perennial forbs to heat shock and smoke treatments in the Chaco Serrano, central Argentina. Austral Ecology, 43(5), 567-577. https://doi.org/10.1111/aec.12593

Argañaraz, J. P., Landi, M. A., Scavuzzo, C. M. y Bellis L. M. (2018). Determining fuel moisture thresholds to assess wildfire hazard: A contribution to an operational early warning system. PLOS ONE, 13(10), e0204889. https://doi.org/10.1371/journal.pone.0204889

Baeza, M. J. y Roy, J. (2008). Germination of an obligate seeder (Ulex parviflorus) and consequences for wildfire management. Forest Ecology and Management, 256(4), 685-693. https://doi.org/10.1016/j. foreco.2008.05.014

Bates, D., Mächler, M., Bolker, B. y Walker, S. (2015). Fitting Linear Mixed-Effects Models Using Ime4. Journal of Statistical Software, 67(1), 1-48. https://doi. org/10.18637/jss.v067.i01

Bolin, J. F. (2009). Heat shock germination responses of three eastern North American temperate species. Castanea, 74(2), 160-167. https://doi.org/10.2179/08010.1

Buhk, C. y Hensen, I. (2006). "Fire seeders" during early post-fire succession and their quantitative importance in south-eastern Spain. Journal of Arid Environments, 66(2), 193-209. https://doi.org/10.1016/j. jaridenv.2005.11.007

Buhk, C., Meyn, A. y Jentsch, A. (2007). The challenge of plant regeneration after fire in the Mediterranean Basin: Scientific gaps in our knowledge on plant strategies and evolution of traits. Plant Ecology, 192(1), 1-19. https://doi.org/10.1007/s11258-006-9224-2

Burger, B. V., Pošta, M., Light, M. E., Kulkarni, M. G., Viviers, M. Z. y Van Staden, J. (2018). More butenolides from plant-derived smoke with germination inhibitory activity against karrikinolide. South African Journal of Botany, 115, 256-263. https://doi.org/10.1016/j. sajb.2018.01.023

Cabido, M. R. y Zak, M. R. (1999). Vegetación del Norte de Córdoba. Secretaría de Agricultura, Ganadería y Recursos Renovables de la provincia de Córdoba y Agencia Córdoba Ambiente, Córdoba, Argentina.

Cáceres, N., Imhof, L., Suárez, M., Hick, E. C. y Galetto, L. (2018). Assessing native germplasm for extensive green roof systems of semiarid regions. Ornamental Horticulture, 24(4), 466-476. https://doi.org/10.14295/ oh.v24i4.1225

Carbone, L. M., Aguirre-Acosta, N., Tavella, J. y Aguilar, R.
(2017). Cambios florísticos inducidos por la frecuencia de fuego en el Chaco Serrano. Boletín de la Sociedad Argentina de Botánica, 52(4), 753-778. https://doi. org/10.31055/1851.2372.v52.n4.18861

Capitanelli, C. (1979). Clima. En: J. B. Vázquez, R. A. Miatello y M. E. Roqué (Eds.), Geografía física de la provincia de Córdoba (45-138). Boldt.

Casal, M., Prado, S., Reyes, O. y Rivas, M. (1 de junio de 2020). Efectos del fuego sobre la germinación de varias especies leguminosas arbustivas. III Congreso Forestal Español. http://secforestales. org/publicaciones/index.php/congresos_forestales/ article/view/15864/15707

Chou, Y., Cox, R. D. y Wester, D. B. (2012). Smoke Water and Heat Shock Influence Germination of Shortgrass Prairie Species. Rangeland Ecology \& Management, 65(3), 260-267. https://doi.org/10.2111/ REM-D-11-00093.1

Co du Toit, J., Van den Berg L. y O'Connor T. G. (2015). Fire effects on vegetation in a grassy dwarf shrubland at a site in the eastern Karoo, South Africa, African Journal of Range \& Forage Science, 32(1), 13-20. https://doi.org/10.2989/10220119.2014.913077

Di Rienzo, J. A., Casanoves, F., Balzarini, M. G., Gonzalez, L., Tablada, M. y Robledo, C. W. (2020). InfoStat versión 2020. Centro de Transferencia InfoStat, FCA, Universidad Nacional de Córdoba, Argentina. http:// www.infostat.com.ar

Downes, K. S., Lamont, B. B., Light M. E. y van Staden J. (2010). The fire ephemeral Tersonia cyathiflora (Gyrostemonaceae) germinates in response to smoke but not the butenolide 3-methyl-2H-furo[2,3-c]pyran2-one. Annals of Botany, 106, 381-384. https://doi. org/10.1093/aob/mcq118

Ernst, W. H. O., Kuiters, A. T. y Tolsma, D. J. (1991). Dormancy of annual and perennial grasses from a savanna of southeastern Botswana. Acta Oecológica, 12, 727-739.

Farías, L., López-Mársico, L. y Rodríguez C. (2016). Efecto del calor y el humo sobre la germinación de especies de pastizales de las Sierras del Este, Uruguay. https:// www.researchgate.net/publication/308963948_ Efecto_del_calor_y_el_humo_sobre_la_germinacion_ de_especies_de_pastizales_de_Sierras_del_Este_ Uruguay

Ferri, R., Ceballos, M., Vischi, N., Heredia, E. y Oggero, A. (2009). Banco de semillas de un relicto de Espinal (Córdoba, Argentina). IHERINGIA, Sér. Bot., 64(1), 93-100. https://isb.emnuvens.com.br/iheringia/article/ view/139

Fuentes, E., Carreras, M. E., Martinat, J. E., Jewsbury, G., Loyola, M. J. y Carbone, L. (4 al 10-10-2010). Especies de valor forrajero en flora serrana posfuego de Córdoba -Argentina. En X Congreso Latinoamericano 
de Botánica, "Conservación y uso sustentable de la flora nativa latinoamericana" (78). La Serena, Chile: Asociación Latinoamericana de Botánica.

Fuentes, E., Carreras, M. E., Carbone, L. M., Jewsbury, G., Loyola, M. J. y Martinat, J. E. (2011). Especies nativas de las Sierras Chicas (Córdoba, Argentina) con estrategias de regeneración post-fuego. Boletín de la Sociedad Argentina de Botánica, 46(S), 192.

Funes, G., Díaz, S. y Venier, P. (2009). La temperatura como principal determinante de la germinación en especies del Chaco seco de Argentina. Ecología Austral, 19(2), 129-138. http://ojs.ecologiaaustral.com. ar/index.php/Ecologia_Austral/article/view/1359/725

Giambastiani, G. (2019). Establecimiento de cultivos estivales. http://agro.unc.edu.ar/ ceryol/documentos/ ecofisiologia/establecimiento.pdf

Giorgis, M. A., Cingolani A. M. y Cabido, M. (2013). El efecto del fuego y las características topográficas sobre la vegetación y las propiedades del suelo en la zona de transición entre bosques y pastizales de las sierras de Córdoba, Argentina. Boletín de la Sociedad Argentina de Botánica, 48(3-4), 493-513. https://doi. org/10.31055/1851.2372.v48.n3-4.7555

Giorgis, M. A., Cingolani, A. M., Chiarini, F., Chiapella, J., Barboza, G., Ariza Espinar, L., Morero, R., Gurvich, D. E., Tecco, P. A., Subils, R. y Cabido, M. (2011). Composición florística del Bosque Chaqueño Serrano de la provincia de Córdoba, Argentina. Kurtziana, 36(1), 9-43.

Haferkamp, M. R. y Jordan, G. L. (1977). The effect of selected presowing seed treatments on germination of Lehmann lovegrass seeds. Journal of Range Management, 30(2), 151-153. https://doi. org/10.2307/3897760

Halvorson, W. L. y Guertin, P. (2003). Factsheet for Eragrostis lehmanniana Nees. https://www.feedipedia. org/node/435

Hanley, M. E., Unna, J. y Darvill, B. (2003). Seed size and germination response: a relationship for fire-following plant species exposed to thermal shock. Oecología, 134(1), 18-22. https://doi.org/10.1007/s00442-0021094-2

Iglesia Rodríguez, A. (2010). Efecto del fuego sobre la germinación y el banco de semillas edáfico de Ericáceas de Galicia. Tesis doctoral, Universidad de Santiago de Compostela, Lugo, España.

International Seed Testing Association (ISTA) (2019). International Rules for Seed Testing. Bassersdorf, Switzerland.

Jewsbury, G., Loyola, M. J., Carbone, L. M., Carreras, M. E., Pons, S. M., Martinat, J. E., Castillo Moine, M. y Fuentes, E. (2016). Pastos Posfuego de las Sierras de Córdoba. Guía de Campo. Ecoval.
Keeley, J. E. y Pausas, J. G. (2018). Evolution of 'smoke' induced seed germination in pyroendemic plants. South African Journal of Botany, 115, 251-255. https:// doi.org/10.1016/j.sajb.2016.07.012

Keith, D. A., McCaw, W. L. y Whelan, R. J. (2002). Fire regimes in Australian heathlands and their effects on plants and animals. In R.A. Bradstock, J.E. Williams, A.M. Gill (Eds.), Flammable Australia: the fire regimes and biodiversity of a continent (199-237). Cambridge University Press.

Kunst, C. R., Bravo, S. y Panigatti, J. L. (2003). Fuego en los ecosistemas argentinos. INTA.

Lamont, B. B. y Downes, K. (2011). Fire-stimulated flowering among resprouters and geophytes in Australia and South Africa. Plant Ecology, 212, 21112125. https://doi.org/10.1007/s11258-011-9987-y

Lupi, A. M. (2015). El fuego: una herramienta de manejo o un arma contra el suelo. https://www.produccionanimal.com.ar/produccion_y_manejo_pasturas/uso_ del_fuego/27-herramienta_de_manejo.pdf

Martinat, J. E. (2012). Efecto del choque térmico simulando la acción del fuego en la germinación de Fabáceas y Poáceas forrajeras de las Sierras Chicas de Córdoba. Tesis de Maestría, Facultad de Ciencias Agropecuarias, Universidad Nacional de Córdoba, Argentina.

Martinat, J. E., Aguirre Juarez, J. I., Dellarrosa, F. J., Barrionuevo, A. E. y Fuentes E. (3-11-2015). Efecto del choque térmico en la germinación de Bouteloua curtipendula (Michx.) Torr. var. caespitosa Gould \& Kapadia, pasto forrajero. En S. Ledesma y C. Giuliano (Eds.), VI Jornadas Integradas de Investigación y Extensión de la FCA (274). Córdoba, Argentina: Universidad Nacional de Córdoba.

Martinat, J. E., Marinsaldi, M., Foruria, D. V., Porciani, N. Y. y Dellarossa, F. J. (2017). Germinación de cariópsides de Eustachys retusa (Lag.) Kunth y Gouinia latifolia (Griseb.) Vasey (Poaceae), forrajeras expuestas a altas temperaturas. Boletín de la Sociedad Argentina de Botánica, 52(S), 202. https://botanicaargentina. org.ar/wp-content/uploads/2017/09/SAB-2018-1.pdf

Martinat, J. E., Vargas, L., Pons, S. M., Reyna, M. E. y Gil, S. P. (20-11-2019). Promover Eustachys retusa (Lag.) Kunth y Schizachyrium condensatum (Kunth) Nees, nativas de las sierras de Córdoba, con fines de uso ornamental en xerojardinería. En M. A. Cicler y A. C. Giuliano, VIII Jornadas Integradas de Investigación, Extensión y Enseñanza de la FCA (151). Córdoba, Argentina: Universidad Nacional de Córdoba.

Moreira, B., Tormo, J., Estrelles, E. y Pausas, J. G. (2010). Disentangling the role of heat and smoke as germination cues in Mediterranean Basin flora. Annals of Botany, 105(4), 627-635. https://doi.org/10.1093/ aob/mcq017 
Overbeck, G. E., Müller, S. C., Pillar, V. D. y Pfadenhauer, J. (2006). No heat-stimulated germination found in herbaceous species from burned subtropical grassland. Plant Ecology, 184, 237-243. https://doi. org/10.1007/s11258-005-9068-1

Paula, S. y Pausas, J. G. (2008). Burning seeds: germinative response to heat treatments in relation to resprouting ability. Journal of Ecology, 96(3), 543-552. https://doi.org/10.1111/j.1365-2745.2008.01359.x

Pausas, J. G. y Schwilk, D. (2012). Fire and plant evolution. New Phytologist, 193, 301-303. https://doi. org/10.1111/j.1469-8137.2011.04010.x

Peichoto, M. C. (2010). Revisión taxonómica de las especies del género Schizachyrium (Poaceae: Andropogoneae) de Sudamérica. Candollea, 65(2), 301-346. https://www.ville-ge.ch/cjb/publications/ cando652/C652_301-345.pdf

Preliasco, P. y Miñarro, F. O. (2016). La Ganadería en el Bosque Chaqueño, ¿Amenaza o Parte de la Solución? Informe Ambiental Anual 2016.

Privitello, L. M. J., Orive, J. y Rosa, S. T. (2010). Implantación de especies perennes megatérmicas en un pastizal natural del área medanosa de San Luis, Argentina. Revista Cubana de Ciencia Agrícola, 44(2), 179-184.

Reeder, J. R. (1977). The germination flap in certain Gramineae. Madroño, 24(2), 123-124. https://www. jstor.org/stable/41424096

Ribeiro, L. C., Pedrosa, M. y Borghetti, F. (2013). Heat shock effects on seed germination of five Brazilian savanna species. Plant Biology, 15(1), 152-157. https://doi.org/10.1111/j.1438-8677.2012.00604.x

Ruyle, G. B., Roundy, B. A. y Cox, J. R. (1988). Effects of burning on germinability of Lehmann lovegrass. Journal of Range Management, 41(5), 404-406. https://doi.org/10.2307/3899577

Sánchez de Lorenzo-Cáceres, J. M. (2006). Selección de elementos vegetales para xerojardinería.Jornadas de Jardinería 2006. Universidad Politécnica de Madrid, España: E.U.I.T.A.

Schrauf, G. E., Martino, A., Giavedoni, J. y Pensiero, J.
F. (1998). Efectos genéticos y ambientales sobre el comportamiento germinativo de poblaciones de Moha perenne. Ecología Austral, 8, 49-56. http://ojs. ecologiaaustral.com.ar/index.php/Ecologia_Austral/ article/view/1635/967

Southwest Environmental Information Network (SEINet) Arizona - New México Chapter (2019). Schizachyrium condensatum. http://swbiodiversity.org/seinet/taxa/ index.php?tid=217305

Tavsanoglu, C. (2011). Fire-related cues (heat shock and smoke) and seed germination in a Cistus creticus population in southwestern Turkey. Ekoloji, 79, 99-104. https://www.researchgate.net/publication/269875007_ Fire-Related_Cues_Heat_Shock_and_Smoke_and_ Seed_Germination_in_a_Cistus_creticus_Population_ in_Southwestern_Turkey

Torrella, S. A., Piquer-Rodríguez, M., Levers, C., Ginzburg, R., Gavier-Pizarro, G. y Kuemmerle, T. (2018). Multiscale spatial planning to maintain forest connectivity in the Argentine Chaco in the face of deforestation. Ecology and Society, 23(4), 37. https:// doi.org/10.5751/ES-10546-230437

Veneciano, J. H. (2006). Gramíneas estivales perennes para ambientes semiáridos: características $y$ productividad. Información Técnica de la Estación Experimental Agropecuaria San Luis (Informe № 171). Instituto Nacional de Tecnología Agropecuaria.

Verzino, G., Joseau, J., Dorado, M., Gellert, E., Rodríguez Reartes, S. y Nóbile, R. (2005). Impacto de los incendios sobre la diversidad vegetal, sierras de Córdoba, Argentina. Ecología Aplicada, 4(1-2), 25-34. https://doi.org/10.21704/rea.v4i1-2.294

Zak, M. R., Cabido, M. y Hodgson, J. G. (2004). Do subtropical seasonal forests in the Gran Chaco, Argentina, have a future? Biological Conservation, 120, 589-598. https://doi.org/10.1016/j.biocon.2004.03.034

Zuloaga, F. O., Rúgolo, Z. E. y Anton, A. M. (Eds). (2012). Flora Argentina. Flora Vascular de la República Argentina. Poaceae, Aristidoideae-Panicoideae 3 (I). Gráficamente Ediciones. 